\title{
An Efficient BPSK Modulation and Demodulation Scheme with the Complete Removal of Fading
}

This paper was downloaded from TechRxiv (https://www.techrxiv.org).

\section{LICENSE}

CC BY 4.0

SUBMISSION DATE / POSTED DATE

05-10-2021/12-10-2021

\section{CITATION}

Nguyen, Trang Tien; Ta, Hien; Oh, Hoon (2021): An Efficient BPSK Modulation and Demodulation Scheme with the Complete Removal of Fading. TechRxiv. Preprint. https://doi.org/10.36227/techrxiv.16735312.v1

DOI 


\title{
An Efficient BPSK Modulation and Demodulation Scheme with the Complete Removal of Fading
}

\author{
Trang Tien Nguyen, Hien Q. Ta and Hoon Oh, Member, IEEE
}

\begin{abstract}
A novel non-coherent BPSK modulation and demodulation scheme is proposed to remove both slow and fast fading completely. We formulate a compound function that reflects the different characteristics of both slow and fast fading and prove that the scheme can remove fading from the function completely. The scheme is reified by a simple architecture that can remove slow and fast phase shifts completely from the received signals. It is shown by using analytical model and numerical data that the proposed scheme outperforms other ones significantly under the presence of both slow and fast fading. The analytical results also show that the proposed scheme can work well even with a small sampling rate, resulting in a wireless device with low energy consumption.
\end{abstract}

Index Terms - bit error probability, modulation, demodulation, non-coherent detection, slow fading, fast fading.

\section{INTRODUCTION}

$\mathrm{T}$ HE binary phase shift keying (BPSK) modulation technique is used as a standard in many wireless networks such as WiFi [1], Wireless Sensor Networks [2], NB-IoT and LTE systems [3]. In conventional BPSK schemes, since a receiver sums up the original signal and many fading signals before demodulation, it may demodulate a distorted signal, thereby increasing the possibility of data error. This paper aims at devising a new BPSK modulation and demodulation technique that can remove fading.

Fading can be of two types: slow and fast [4]. The slow fading, caused by reflected signals, results in a slowly varying phase shift and the fast fading, caused by the external interferences from radios using the same frequency bands or the doppler effect, may incur a phase shift that can vary faster than a symbol duration. There are two general detection techniques to improve the phenomenon of fading: coherent and noncoherent [4]. A receiver using coherent detection requires the knowledge of phase shift so that it can remove the phase shift before going into the demodulation process for the received signals. However, the ideal coherent detection is not practical since it is not possible to get the exact phase shift value. Thus, a receiver employs the non-ideal coherent detection that estimates the phase shift by using a preamble [5]. In most cases, a receiver uses the non-coherent detection technique that demodulates signals based on the phase difference of two consecutive symbols. Thus, it works well with slow fading [6][7][8]. However, the previous approaches, using either coherent or non-coherent detections, fail to solve the fast fading that prevails in office or industrial environments [3].
Furthermore, they require a complex receiver architecture which is not preferred for most wireless devices that require low energy consumption.

In this paper, we propose a novel BPSK modulation and demodulation scheme that can remove both slow and fast fading completely. For this, we formulate a compound function that reflects the characteristics of both slow and fast fading and prove that the proposed scheme can remove the fading from the compound function. We compare the proposed scheme with the conventional ones by using analytical model and numerical data in terms of a bit error rate (BER).

\section{BACKGROUND}

In this section, we derive a bit error probability function without considering the channel coding used in some of the previous coherent and non-coherent techniques, and compare them with the ideal coherent receiver under slow and fast fading in terms of BER.

In general, a received BPSK signal $r(t)$ can be represented as a function of time $t$ as follows [9].

$r(t)=\alpha \sqrt{2 E_{b} / T_{b}} \cos \left(2 \pi f_{C} t+(1-n) \pi+\psi+\phi(t)\right)+\eta(t)$,

where $\alpha$ is the fading channel gain based on the Rayleigh fading model and the following exponential distribution $p_{\alpha}(x)$ with common variance $\sigma^{2}[9]$ :

$$
p_{\alpha}(x)=\frac{x}{\sigma^{2}} e^{-x^{2} /\left(2 \sigma^{2}\right)}, \text { for } x>0
$$

$n \in\{0,1\}$ is the BPSK modulated phase, $E_{b}, T_{b}$ and $f_{c}$ indicate energy per bit, bit duration and carrier wave frequency, respectively, $\psi$ is the slow fading phase shift, which remains constant during one bit duration, $\phi(t)$ is the fast fading phase shift, which varies faster than one bit duration, and $\eta(t)$ indicates the noise with the Gaussian distribution of mean 0 and variance $N / 2, N$ is energy of noise. Both $\psi$ and and $\phi(t)$ has uniform distribution with the following probability density function (PDF) [9]:

$$
p_{\phi / \psi}(y)=\frac{1}{2 \pi}, \text { for } y \in[-\pi, \pi]
$$

In the traditional coherent receiver, the received signal goes through the down convert with a local carrier (LC) frequency, 
$f_{L C}$. Hence, the received signal after passing the down convert can be formulated as follows:

$$
\begin{aligned}
r(t) \cos \left(2 \pi f_{L C} t\right)= & \alpha \sqrt{2 E_{b} / T_{b}} \cos \left(2 \pi f_{C} t+(1-n) \pi+\psi+\right. \\
& \phi(t)) \cos \left(2 \pi f_{L C} t\right)+\eta(t) \cos \left(2 \pi f_{L C} t\right) .
\end{aligned}
$$

The signal continues to go through the low-pass filter and the integration component, resulting in the following signal:

$$
\begin{aligned}
r(t)= & \frac{\alpha}{2} \sqrt{2 E_{b} / T_{b}} \int_{0}^{T_{b}} \cos \left(2 \pi\left(f_{C}-f_{L C}\right) t+(1-n) \pi+\psi+\right. \\
& \phi(t)) d t+\int_{0}^{T_{b}} \eta(t) \cos \left(2 \pi f_{L C} t\right) d t
\end{aligned}
$$

where, $\cos ((1-n) \pi)$ becomes the value of \pm 1 and for simplicity, the residual frequency component of $2 \pi\left(f_{C}-f_{L C}\right) t$ can be combined into $\phi(t)$ to yield:

$$
\begin{aligned}
r(t)= & \frac{\alpha}{2} \sqrt{2 E_{b} / T_{b}} \int_{0}^{T_{b}} \pm \cos (\psi+\phi(t)) d t \\
& +\int_{0}^{T_{b}} \eta(t) \cos \left(2 \pi f_{C} t\right) d t \\
= & \pm \frac{\alpha}{2} \sqrt{2 E_{b} / T_{b}} \times T_{b}\left[\cos (\psi) \int_{-\pi}^{\pi} \cos (y) p_{\phi}(y) d y\right. \\
& \left.\quad-\sin (\psi) \int_{-\pi}^{\pi} \sin (y) p_{\phi}(y) d y\right]+ \\
& \int_{0}^{T_{b}} \eta(t) \cos \left(2 \pi f_{L C} t\right) d t \\
= & \int_{0}^{T_{b}} \eta(t) \cos \left(2 \pi f_{L C} t\right) d t,
\end{aligned}
$$

which is derived because $\int_{-\pi}^{\pi} \cos (y) p_{\phi}(y) d y=0$ and $\int_{-\pi}^{\pi} \sin (y) p_{\phi}(y) d y=0$.

Remark 1: Observing the only noise signal in (7), one can obtain the bit error probability of 0.5 regardless of signal-tonoise ratio (SNR), $E_{b} / N$. This indicates that the traditional coherent or non-coherent receiver is completely failed under fast fading.

Next, we consider the case of only slow fading, i.e. $\phi(t)=0$, where (6) reduces to

$r(t)= \pm \frac{\alpha}{2} \sqrt{2 E_{b} T_{b}} \cos (\psi)+\int_{0}^{T_{b}} \eta(t) \cos \left(2 \pi f_{L C} t\right) d t$.

Since the term $\int_{0}^{T_{b}} \eta(t) \cos \left(2 \pi\left(f_{L C}\right) t\right) d t$ has Guassian distribution with mean 0 and vairance $N T_{b} / 4$, the bit error probability can be obtained by

$$
\begin{aligned}
P_{e}(\alpha) & =Q\left(\frac{\frac{\alpha}{2} \sqrt{2 E_{b} T_{b}} \cos (\psi)}{\sqrt{N T_{b} / 4}}\right) \\
& =Q\left(\alpha \sqrt{2|\cos (\psi)|^{2} E_{b} / N}\right) .
\end{aligned}
$$

\section{A. Ideal Coherent Detection}

The ideal coherent detection assumes that the phase shifts can be known perfectly and removed at the receiver, i.e. $\psi=0$ and $\phi(t)=0$. Then, the average bit error probability, averaging (9) when $\psi=0$ with respect to $\alpha$, for the ideal coherent detection is given by

$$
\begin{aligned}
P_{e, i c o h} & =\int_{0}^{\infty} Q\left(x \sqrt{2 E_{b} / N}\right) p_{\alpha}(x) d x \\
& =\frac{1}{2}\left[1-\sqrt{\frac{2 \sigma^{2} E_{b} / N}{1+2 \sigma^{2} E_{b} / N}}\right],
\end{aligned}
$$

which is derived from [Equation 13.3-7, 9].

\section{B. Non-ideal Coherent Detection}

The non-ideal coherent detection uses the coherent receiver with unknown phase shift $\psi$ in slow fading. Then, $P_{e, n c o h}$ as the average bit error probability for the non-ideal coherent detection scheme can be obtained from (9) and (10),

$$
P_{e, n c o h}=\frac{1}{2}\left[1-\int_{-\pi}^{\pi} \sqrt{\frac{|\cos (y)|^{2} 2 \sigma^{2} E_{b} / N}{1+|\cos (y)|^{2} 2 \sigma^{2} E_{b} / N}} p_{\psi}(y) d y\right] .
$$

\section{Differential Modulation}

In this subsection, we further consider the differential modulation scheme, which is the well-known method to remove slow fading. Then, the average bit error probability of the differential modulation scheme can be obtained by [Equation 13.3-10, 9]

$$
P_{e, d m}=\frac{0.5}{1+\sigma^{2} E_{b} / N}
$$

\section{THE PROPOSED APPROACH}

In this section, we present a non-coherent BPSK modulation and demodulation scheme and compare the proposed scheme and the previous ones analytically in terms of BER.

\section{A. BPSK modulation and demodulation scheme}

A transmitted signal $s(t)$ at time $t$ is represented as:

$s(t)=\sqrt{E_{b} / T_{b}} \sin \left(2 \pi f_{C} t+\varphi_{1}\right)+\sqrt{E_{b} / T_{b}} \cos \left(2 \pi f_{C} t+\varphi_{2}\right)$,

where $\left|\varphi_{1}-\varphi_{2}\right|$ is given $3 \pi / 2$ for bit ' 0 ' and $\pi / 2$ for bit ' 1 ' approximately. Refer to the transmitter architecture in Fig 1.

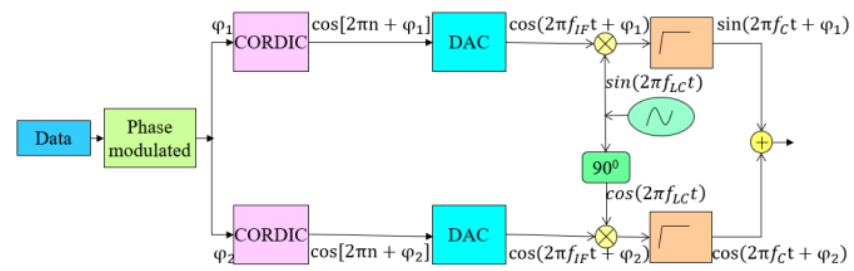

Fig. 1. The transmitter architecture corresponding to the proposed BPSK modulation and demodulation scheme.

A received signal $r(t)$ at time $t$ is represented as:

$$
\begin{aligned}
r(t)= & \alpha \sqrt{E_{b} / T_{b}} \sin \left(2 \pi f_{C} t+\varphi_{1}+\psi+\phi(t)\right) \\
& +\alpha \sqrt{E_{b} / T_{b}} \cos \left(2 \pi f_{C} t+\varphi_{2}+\psi+\phi(t)\right)+\eta(t)
\end{aligned}
$$


A receiver architecture that accounts for the proposed scheme is illustrated in Fig. 2. The received signal is separated into two signals by the down convert with local carrier (LC) frequency, one going through the in-phase (I) channel and another through quadratic-phase $(\mathrm{Q})$ channel. Those signals are transformed to intermediate frequencies (IFs) by passing a lowpass filter, and then converted to digital signals via the ADC components. Then, after taking the square and integral of those digital signals, they are summed, thereby removing the phase shifts completely and leaving only the modulated phase of the BPSK signal.

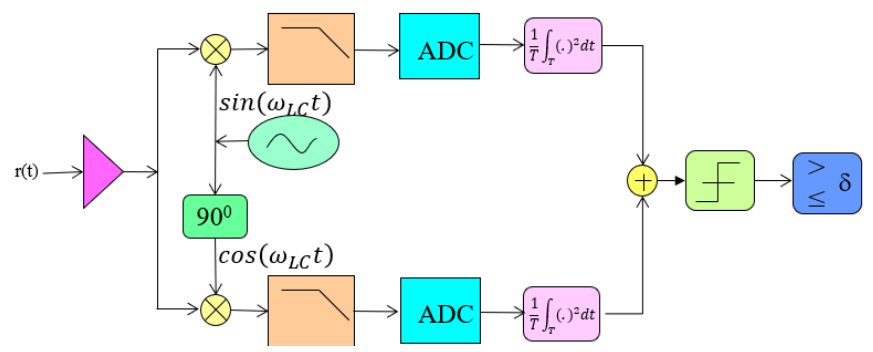

Fig. 2. The receiver architecture corresponding to the proposed BPSK modulation and demodulation scheme.

Let us describe the process formally. After going through the down convert with a local carrier (LC) frequency and the low pass filters, I and Q signals can be expressed as follows:

$$
\begin{aligned}
& r(t) \cos \left(2 \pi f_{L C} t\right) \\
& =\frac{\alpha}{2} \sqrt{E_{b} / T_{b}} \sin \left(2 \pi\left(f_{C}-f_{L C}\right) t+\varphi_{1}+\psi+\phi(t)\right) \\
& \quad+\frac{\alpha}{2} \sqrt{E_{b} / T_{b}} \cos \left(2 \pi\left(f_{C}-f_{L C}\right) t+\varphi_{2}+\psi+\phi(t)\right) \\
& \quad+\eta(t) \cos \left(2 \pi f_{L C} t\right) \\
& =\alpha \sqrt{E_{b} / T_{b}} \sin \left(2 \pi\left(f_{C}-f_{L C}\right) t+\psi+\phi(t)+\frac{\varphi_{1}+\varphi_{2}}{2}+\frac{\pi}{4}\right) \\
& \quad \times \cos \left(\frac{\varphi_{1}-\varphi_{2}}{2}-\frac{\pi}{4}\right)+\eta(t) \cos \left(2 \pi f_{L C} t\right),
\end{aligned}
$$

and

$$
\begin{aligned}
r & (t) \sin \left(2 \pi f_{L C} t\right) \\
= & \frac{\alpha}{2} \sqrt{E_{b} / T_{b}} \cos \left(2 \pi\left(f_{C}-f_{L C}\right) t+\varphi_{1}+\psi+\phi(t)\right) \\
& -\frac{\alpha}{2} \sqrt{E_{b} / T_{b}} \sin \left(2 \pi\left(f_{C}-f_{L C}\right) t+\varphi_{2}+\psi+\phi(t)\right) \\
& +\eta(t) \sin \left(2 \pi f_{L C} t\right) \\
= & \alpha \sqrt{E_{b} / T_{b}} \cos \left(2 \pi\left(f_{C}-f_{L C}\right) t+\psi+\phi(t)+\frac{\varphi_{1}+\varphi_{2}}{2}+\frac{\pi}{4}\right) \\
& \times \cos \left(\frac{\varphi_{1}-\varphi_{2}}{2}-\frac{\pi}{4}\right)+\eta(t) \sin \left(2 \pi f_{L C} t\right) .
\end{aligned}
$$

After going through the ADC components, the I and Q digital signals are squared, integrated, and summed to become:

$$
\begin{aligned}
& \frac{1}{T_{b}} \int_{0}^{T_{b}}\left[\left(r(t) \cos \left(2 \pi f_{L C} t\right)\right)^{2}+\left(r(t) \sin \left(2 \pi f_{L C} t\right)\right)^{2}\right] d t \\
& \approx \frac{\alpha^{2} E_{b}}{T_{b}}\left(\cos \left(\frac{\varphi_{1}-\varphi_{2}}{2}-\frac{\pi}{4}\right)\right)^{2}+\frac{1}{T_{b}} \int_{0}^{T_{b}}(\eta(t))^{2} d t
\end{aligned}
$$

where the cross terms between the noise and signal are negligible and hence, can be ignored, resulting in (17). Finally, (17) is compared with a threshold, denoted by $\delta$, to decide bit ' 0 ' or ' 1 '.

Remark 2: One can observe from (17) that the received signal after processing becomes the clean signal that includes only the modulated phase while the phase shifts of both slow and fast fading are completely removed. This confirms the robustness of our proposed scheme to remove fading completely.

\section{B. Error Probability}

In this subsection, the bit error probability of the proposed scheme is examined analytically. The detection threshold $\delta$ is determined under the condition that the error probability when bit ' 0 ' or ' 1 ' is sent is equiprobable. Let $K$ and $\eta$ ' denote the number of samples taken during one bit duration, and the approximated noise as the second term in the right-hand side of (17). Then, $\eta^{\prime}$ can be rewritten as follows:

$$
\eta^{\prime}=\frac{1}{K} \sum_{i=1}^{K}(\eta[i])^{2} .
$$

Equation (18) corresponds to the Gaussian distribution with mean $N / 2$ and variance $N^{2} /(2 K)$ approximately [Equation $2.5-3,9]$.

When bit ' 0 ' is transmitted, the bit error probability is given by

$$
\begin{aligned}
\operatorname{Pr}(\hat{b}=1 \mid b=0) & =\operatorname{Pr}\left(\eta^{\prime}>\delta \mid b=0\right) \\
& =Q((\delta / N-0.5) \sqrt{2 K}),
\end{aligned}
$$

and when bit ' 1 ' is transmitted, it is given by

$$
\begin{aligned}
& \operatorname{Pr}(\hat{b}=0 \mid b=1) \\
& =\operatorname{Pr}\left(\alpha^{2} E_{b} / K+\eta^{\prime} \leq \delta \mid b=1\right) \\
& =\int_{0}^{\infty} Q\left(\left(x^{2} E_{b} /(N * K)+0.5-\delta / N\right) \sqrt{2 K}\right) p_{\alpha}(x) d x .
\end{aligned}
$$

According to the requirement of the equiprobable error probability, the threshold $\delta$ is found by equating (19) and (20), i.e., $\operatorname{Pr}(\hat{b}=1 \mid b=0)=\operatorname{Pr}(\hat{b}=0 \mid b=1)$. Then, we can obtain $\delta$ by performing numerical computation, and then obtain the error probability using $\delta$ from (19).

\section{NUMERICAL RESULTS}

By resorting to Matlab, we compare the proposed noncoherent scheme with direct demodulation (DirNonCoh) with the conventional non-coherent scheme with differential demodulation (DiffNonCoh), and two coherent schemes, ideal coherent and non-ideal coherent schemes, named IdealCoh and NonIdealCoh, respectively. 


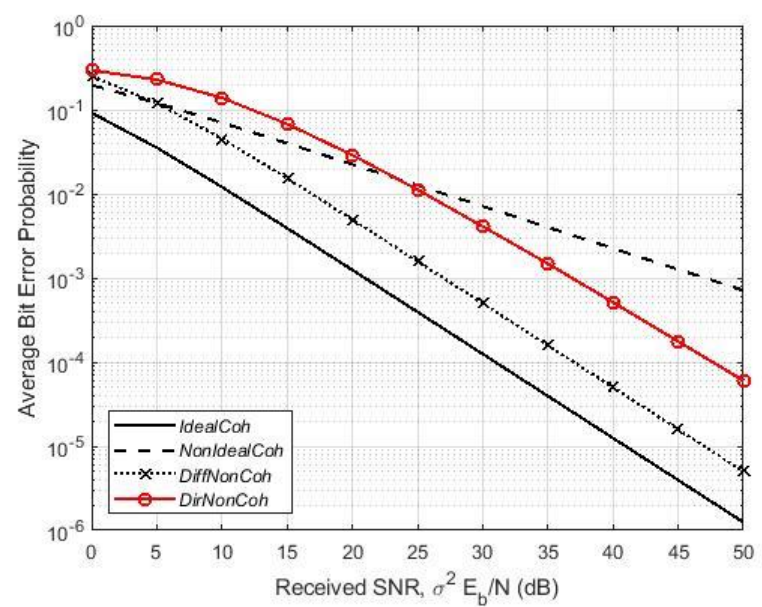

Fig. 3. The bit error probability versus the received $S N R$, $\sigma^{2} E_{b} / N$, under only slow fading $(K=20)$.

Fig. 3 shows the bit error probability versus the received signal-to-noise ratio, $\sigma^{2} E_{b} / N$, only under slow fading. It is shown that the proposed scheme, DirNonCoh is worse than NonIdealCoh in bit error probability with low SNRs; however, they start crossing at SNR of $25 \mathrm{~dB}$. Note that DirNonCoh can achieve BER of $10^{-4}$ required in the wireless network standards with SNR of $47 \mathrm{~dB}$ which is said to be reasonable in real environment [4][9]. Comparing with DiffNonCoh, DirNonCoh needs additional $10 \mathrm{~dB}$ to achieve the BER of $10^{-4}$; however, our approach DirNonCoh is still favored in the slow fading communication technologies since it detects bit directly while DiffNonCoh detects a bit by comparing the differential phase of two consecutive symbols with their coherent times.

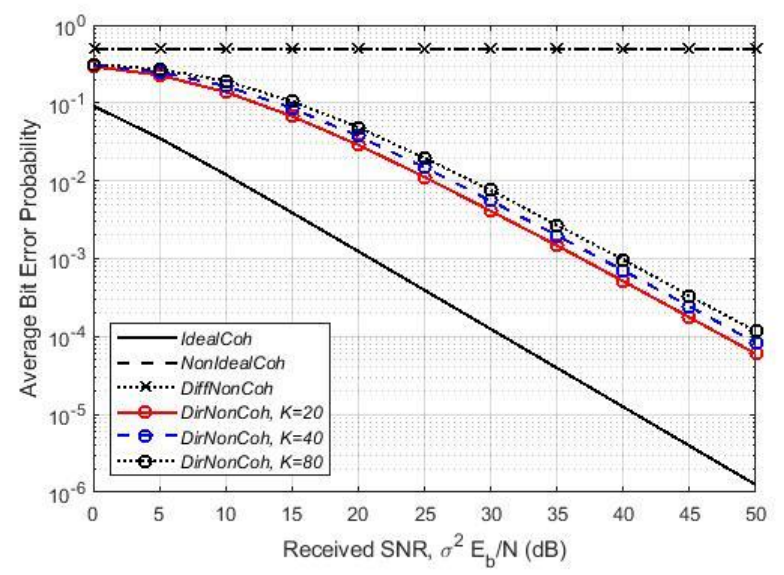

Fig. 4. The error probability versus the received SNR, $\sigma^{2} E_{b} / N$, under both slow and fast fading with different $K$ values.

Fig. 4 shows the bit error probability versus the received signal-to-noise ratio, $\sigma^{2} E_{b} / N$, under both slow and fast fading. One can observe that NonIdealCoh and DiffNonCoh sustains the BER of 0.5 constantly regardless of SNR. This is because the fast fading tends to make the estimation of the phase shift wrong. On the contrary, the proposed DirNonCoh works regardless of fading because it removes phase shift completely before demodulation. Interestingly, the proposed scheme achieves the slightly lower BER by decreasing $K$ (the number of samples taken during one bit duration). In reality, the low $K$ is preferred for most wireless devices due to complexity and energy consumption.

\section{CONCLUSION}

In this paper, a novel non-coherent modulation and demodulation scheme was proposed to remove both slow and fast fading completely. The scheme was reified by a simple receiver architecture that illustrates its operation, and then was proven for its correctness by mathematical analysis and numerical data. According to the results, it was shown that the proposed scheme could achieve the required wireless standard BER of $10^{-4}$ in the presence of both slow and fast fading while other schemes achieve the standard BER only for slow fading. Furthermore, since the proposed scheme achieves the standard BER with much smaller number of samples, it can work with much low complexity and energy consumption.

\section{REFERENCES}

[1] L. Verma, M. Fakharzadeh, and S. Choi, "Wifi on steroids: 802.11 ac and 802.11 ad," IEEE Wireless Communications, vol. 20, no. 6, pp. 30-35, 2013.

[2] "Approved IEEE Draft Amendment to IEEE Standard for Information Technology-Telecommunications and Information Exchange Between Systems-Part 15.4:Wireless Medium Access Control (MAC) and Physical Layer (PHY) Specifications for LowRate Wireless Personal Are," IEEE Approv. Std P802.15.4a/D7, Jan 2007, 2007.

O. Liberg, M. Sundberg, E. Wang, J. Bergman, and J. Sachs, Cellular Internet of things: technologies, standards, and performance. Academic Press, 2017.

D. Tse and P. Viswanath, "Fundamentals of Wireless Communication12," Notes, p. 583, 2004.

T. T. Nguyen and H. Oh, "A Receiver for Resource-Constrained Wireless Sensor Devices to Remove the Effect of Multipath Fading," IEEE Trans. Ind. Electron., vol. 65, no. 7, pp. 6009-6016, 2018.

S. J. Henely and M. D. Walby, "Differential phase-shift keying demodulator." Google Patents, 1990.

D. Warrier and U. Madhow, "Spectrally efficient noncoherent communication," IEEE Trans. Inf. Theory, vol. 48, no. 3, pp. 651668, 2002.

[8] D. Divsalar and M. K. Simon, "Multiple-symbol differential detection of MPSK," IEEE Trans. Commun., vol. 38, no. 3, pp. 300308, 1990.

[9] J. G. Proakis, Digital Communications. McGraw-Hill, 2001. 